\title{
Blefaroespasmo essencial: revisão da literatura
}

\author{
Essential blepharospasm: literature review \\ André Gustavo Bombana Nicoletti ${ }^{1}$, Lísia Aoki ${ }^{1}$, Tatiana Rizkallah Nahas ${ }^{2}$, Suzana Matayoshi ${ }^{1}$
}

\section{RESUMO}

O blefaroespasmo essencial é uma distonia focal caracterizada por contrações involuntárias, espasmódicas e bilaterais dos músculos orbicular da pálpebra, corrugador, prócerus e depressor do supercílio. Trata-se de uma doença rara, cujo diagnóstico é frequentemente tardio, o que permite a progressão dos sintomas até quadros de cegueira funcional. Nesse estudo, por meio de revisão da literatura, descreve-se as principais características da doença, incluindo os seguintes aspectos: histórico, epidemiologia, etiologia, quadro clínico, diagnóstico diferencial e tratamento.

Descritores: Blefarospasmo/etiologia; Blefarospasmo/diagnóstico; Blefarospasmo/epidemiologia; Blefarospasmo/quimioterapia; Doenças palpebrais; Diagnóstico diferencial

\section{ABSTRACT}

Essential blepharospasm is a focal distonia characterized by involuntary, spasmodic, bilateral contractions of eyelid protractors. This is a rare disease, which diagnosis is frequently late and symptoms may progress until functional blindness. In this article, we perform a review and describe the most important features of the disease, including historical aspects, epidemiology, etiology, clinical findings, differential diagnosis and treatment.

Keywords: Blepharospasms/etiology; Blepharospasms/diagnosis; Blepharospasms/epidemiology; Blepharospasms/drug therapy; Eyelid diseases; Diagnosis, differential

\section{INTRODUÇÃO}

0 blefaroespasmo essencial é uma distonia focal caracterizada por contrações involuntárias, espasmódicas e bilaterais dos músculos orbicular da pálpebra (porção orbital e palpebral pré-septal e pré-tarsal), corrugador, prócerus e depressor do supercílio(1). Inicialmente, os sintomas costumam ser intermitentes, com períodos de remissão e exacerbação. Porém, com a progressão da doença, há grave comprometimento da qualidade de vida, evoluindo, em alguns casos, para cegueira funcional ${ }^{(2)}$. Dessa forma, o impacto psicológico da doença pode ser muito grande - sintomas de depressão, ansiedade, obsessivo-compulsivos frequentemente estão associados aos espasmos ${ }^{(2-3)}$.

No entanto, por ser uma doença rara, muitos médicos não a conhecem e encaminham o paciente para avaliação psiquiátrica. Em média, nos Estados Unidos da América, os doentes visitam 4 médicos até que o diagnóstico correto seja estabelecido(2).

Com o intuito de alertar os colegas sobre essa doença e portanto permitir uma abordagem adequada desses pacientes, realizou-se extensa revisão da Literatura, enfocando os principais aspectos relacionados ao histórico da doença, epidemiologia, etiologia, quadro clínico, diagnóstico diferencial e tratamento.

\section{HISTÓRICO}

Até a metade do século XX, poucos avanços tinham sido feitos para a compreensão e o tratamento do blefaroespasmo e das distonias associadas e a maioria dos pacientes ainda era considerada mentalmente instável.

O primeiro registro do blefaroespasmo na Literatura Médica foi em 1870, quando *Talkow, citado por Anderson ${ }^{(2)}$, descreveu pacientes com espasmos faciais e palpebrais.

Henderson ${ }^{(1)}$, baseado nos registros da Clínica Mayo, descreve o tratamento que vinha sendo oferecido aos pacientes na primeira metade do século XX. Diversas medicações foram utilizadas de forma empírica, tais como antiparkinsonianos, hipnóticos e anfetaminas, com resultados pouco duradouros e acompanhados por diversos efeitos colaterais. Alguns pacientes também foram submetidos a sessões de psicoterapia, sem resultados práticos. Os procedimentos cirúrgicos se limitavam a injeções de álcool, eletrocoagulação e ressecções cirúrgicas do nervo facial e músculo orbicular. Entretanto, os pacientes sofriam com altas taxas de recorrência e importantes efeitos colaterais decorrentes da disfunção desse nervo, como perda da expressão e/ou movimentação facial, de tal forma que o tratamento podia ser tão debilitante quanto à doença.

\section{EPIDEMIOLOGIA}

Poucos são os estudos epidemiológicos disponíveis sobre blefaroespasmo essencial. Em uma revisão da literatura, Defazio e Livrea(4) relacionaram cinco estudos populacionais que estimaram que a prevalência de blefaroespasmo varia entre $16 \mathrm{e}$ 133 casos por milhão. Em uma avaliação mais recente, Cossu et al.(5) estimaram a prevalência do blefaroespasmo essencial na ilha de Sardenha em 32,2 casos por milhão de habitantes.

* Talkow (1870) apud ${ }^{(2)}$
Trabalho realizado na Clínica Oftalmológica do Hospital das Clínicas da FMUSP. ${ }^{1}$ Médico, Departamento de Plástica Ocular, Hospital das Clínicas, Faculdade de Medicina, Universidade de São Paulo - USP - São Paulo (SP), Brasil. Paulo (SP), Brasil.

A pesquisa foi desenvolvida sem fontes de auxílio.

Endereço para correspondência: André G. B. Nicoletti. Rua 1ํ de Maio, 184 - Apto. 60 Santo André (SP) - CEP 09015-030

E-mail: agbnicoletti@yahoo.com.br

Recebido para publicação em 20.04.2009

Aprovação em 07.06.2010 
A incidência de blefaroespasmo essencial foi calculada em apenas dois estudos do mesmo grupo. Entre os anos de 1950 a 1982, Nutt et al.(6) estimaram a incidência anual da doença em Olmsted County, Minnesota (Estados Unidos da América) em 0,47 casos/100.000 habitantes. Posteriormente, Bradley et al. ${ }^{(7)}$ detectaram a ocorrência anual de 1,2 casos/100.000 habitantes na mesma população, entre os anos de 1976 a 1995.

A idade média do início dos sintomas de blefaroespasmo é de 56 anos, conforme o registro inicial de Henderson ${ }^{(1)}$. Anderson et al. ${ }^{(2)}$ avaliaram retrospectivamente através de questionários, 1.653 pacientes com blefaroespasmo, sendo o maior grupo de doentes já estudado. Esses autores encontraram uma idade média de 55,5 anos.

Há predominância do sexo feminino nessa doença, e a proporção de casos femininos/masculinos varia entre 2 e 3/1 na maioria dos estudos realizados $2,2,8)$.

\section{ETIOLOGIA}

A etiologia do blefaroespasmo essencial permanece desconhecida. Anderson et al. (2) afirmam que os inúmeros estudos, relatos e a observação de pacientes indicam que essa doença tem uma origem multifatorial. Esses autores acreditam na inexistência de um centro específico causador da doença; essa ocorreria por um circuito superativado, que não estaria sendo modulado. Esse circuito se constitui em uma via aferente, um centro de controle e uma via eferente. A via aferente recebe estímulos diversos, como luz, irritação ocular, dor, estresse, fatores emocionais, etc. Esses estímulos são transmitidos a um centro de controle, ainda não identificado, mas provavelmente dentro ou próximo dos gânglios da base. Mesencéfalo ou tronco cerebral seriam outras localizações possíveis. A via eferente é transmitida via núcleo facial, nervo facial e músculos protratores, determinando a contração palpebral. Esse circuito poderia entrar em um ciclo vicioso, no qual ocorrendo uma maior aferência, haveria uma maior eferência. E, quanto mais espasmos, mais estresse, irritação ocular, reiniciando-se o ciclo. O centro de controle, seja por predisposição genética, seja por lesão, seria incapaz de modular este circuito superativado.

McCann et al. ${ }^{(9)}$ observaram que diversos estudos sobre pacientes com blefaroespasmo referem que a maioria desses pacientes apresentam sintomas sensitivos oculares (irritação, olho seco e principalmente fotofobia) e que esses poderiam ser mantidos pelo sistema nervoso simpático, implicado na manutenção de algumas síndromes de dor crônica. Dezenove pacientes foram submetidos a bloqueios simpáticos cervicais com lidocaína e 13 deles $(68,4 \%)$ apresentaram melhora dos sintomas oculares e também 13 pacientes $(68,4 \%)$ passaram a tolerar estímulos luminosos por maiores períodos de tempo. Os autores especularam que o sistema simpático faria parte de uma via que produz fotofobia e causa hiperativação de interneurônios do tronco cerebral que modulam o reflexo do piscar.

Elston et al.(10) avaliaram os registros de prontuários de 170 pacientes com blefaroespasmo e identificaram 69 pacientes $(40,6 \%)$ que apresentaram doenças oculares inicialmente e que foram sucedidas pelos espasmos. Dezenove pacientes foram atendidos com doença ocular unilateral, aguda e dolorosa, com boa resposta ao tratamento. Esses indivíduos geralmente piscavam excessivamente como parte do complexo de sintomas que experimentavam e os espasmos palpebrais se iniciaram em até seis meses após o tratamento em 16 deles. Os autores afirmam que essa estreita relação temporal pode sugerir que quadros oculares ou palpebrais dolorosos podem ter sido responsáveis pelo início da distonia. Outros 50 pacientes foram atendidos inicialmente por doença ocular bilateral, sendo que a maior parte dos casos foi por blefarite ou síndrome do olho seco. O desenvolvimento dos espasmos, nesses casos bilaterais, foi mais tardio, ocorrendo após dois anos em 37 casos (média: 3,7 anos).

Alguns autores aplicaram métodos de imagem, como tomografia por emissão de pósitrons (PET) ${ }^{(11)}$ e ressonância nuclear magnética funcional (fMRI)(12-13) para tentar elucidar os mecanismos envolvidos na fisiopatologia do blefaroespasmo. Schmidt et al. ${ }^{(12)}$ realizaram exames de fMRI em 6 pacientes com a doença e em todos eles puderam correlacionar ativação uni ou bilateral do putâmen com os espasmos palpebrais. Os autores não detectaram qualquer sinal de ativação de gânglios da base ou do tálamo de indivíduos sadios durante contrações voluntárias das pálpebras.

Outra evidência da participação de núcleos da base na fisiopatologia do blefaroespasmo é fornecida pelo relato de Grandas et al. ${ }^{(14)}$, que descrevem o caso de uma paciente que apresentou blefaroespasmo secundário bilateral reflexo após sofrer acidente vascular cerebral, com infarto do corpo estriado direito (envolvia putâmen e núcleo caudado).

Diversos estudos forneceram evidências de que o blefaroespasmo pode se manifestar em famílias ${ }^{(15-17)}$, sendo que os casos familiares variaram entre 12 a 29\%. Defazio et al. ${ }^{(17)}$ descreveram duas famílias italianas com membros acometidos pela doença e concluíram que o padrão de envolvimento sugeria herança autossômica dominante, com baixa penetrância. Esses mesmos autores, em um estudo posterior ${ }^{(16)}$, submeteram a exame neurológico 233 parentes de primeiro grau de $56 \mathrm{pa}$ cientes com blefaroespasmo essencial, ainda que fossem completamente assintomáticos. Entre os 56 pacientes com a doença, 15 (27\%) tinham pelo menos um parente de primeiro grau afetado por alguma distonia de início no adulto, sendo que apenas 5 deles tinham um parente com blefaroespasmo essencial. Os outros 10 pacientes tinham parentes afetados por outras distonias, tais como distonia oromandibular focal, distonia craniocervical segmentar, distonia cervical focal e distonia focal de mãos. Os autores afirmam que essa heterogeneidade do fenótipo da distonia sugere que famílias de pacientes com blefaroespasmo podem ser mais susceptíveis ao desenvolvimento das distonias de início no adulto do que em relação ao blefaroespasmo essencial. Além disso, como cerca de um quarto dos pacientes com espasmos tem parentes de primeiro grau com a doença, os autores concluem que se a história familiar resulta de fatores genéticos, o padrão provável de transmissão é autossômico dominante, com uma penetrância reduzida de cerca de $20 \%$

Muitos pacientes relatam um estresse importante como causa do blefaroespasmo ${ }^{(2)}$. Johnson et al. ${ }^{(18)}$ estudaram pacientes com blefaroespasmo essencial e espasmo hemifacial e os compararam a um grupo controle, em relação à ocorrência de grandes eventos estressantes da vida ("stressful life events"), tais como: morte do cônjuge, infidelidade conjugal, divórcio, incêndio residencial, sérias dificuldades no trabalho e falência. Esses autores não encontraram diferenças estatisticamente significantes entre os grupos em relação ao número de eventos estressantes ocorridos durante a vida, mas observaram que os espasmos faciais começaram dentro de um ano após um desses eventos em 16 de 23 (70\%) participantes do estudo. Além disso, 18 (90\%) em 20 indivíduos tinham tido 2 eventos estressantes separados por um período de um ano ou menos.

Defazio et al. ${ }^{(8)}$, em um estudo multicêntrico de caso-controle, avaliaram a influência do tabagismo e da ingestão de café sobre o risco do desenvolvimento do blefaroespasmo essencial. Empregando questionários, encontraram uma associação inversa significante entre a ocorrência da doença e o consumo de café. Uma xícara de café a mais por dia aumenta a idade de início dos espasmos em 1,7 anos. Os autores também encontraram uma associação inversa com tabagismo, porém não significante quando ajustada pelas variáveis de confusão. 


\section{QUADRO CLÍNICO}

O blefaroespasmo essencial é uma distonia focal caracterizada por contrações involuntárias, espasmódicas e bilaterais dos músculos orbicular da pálpebra (porção orbital e palpebral préseptal e pré-tarsal), corrugador, prócerus e depressor do supercílio(1) (Figura 1).

No início do quadro, os pacientes referem sintomas insidiosos, com aumento da frequência ou duração do ato de piscar ou mesmo dificuldade em manter os olhos abertos. No estudo de Anderson et al. ${ }^{(2)}$, os sintomas iniciais incluíram um aumento da frequência de piscar em $77 \%$ dos casos.

A maioria dos pacientes com blefaroespasmo também apresenta uma produção de lágrimas deficiente $e^{(2,-10)}$, o que tende a causar irritação ocular e consequentemente agravar os espasmos. Elston et al. ${ }^{(10)}$ avaliaram 272 doentes e em 154 indivíduos (57\%) haviam queixas oftalmológicas no início do quadro, incluindo irritação, fotofobia, sensação de olhos secos ou de corpo estranho. McCann et al. ${ }^{(9)}$ observaram que $52,6 \%$ dos pacientes com blefaroespasmo essencial apresentavam ceratopatia ponteada superficial em pelo menos um dos olhos e $95 \%$ dos casos tinham doença da superfície ocular documentada por teste de Schirmer, tempo de rompimento da lágrima e ceratopatia ponteada. Costa et al.(19) avaliaram o filme lacrimal de pacientes com blefaroespasmo e descreveram que todos os pacientes referiram pelo menos algum sintoma de olho seco.

Alguns fatores podem agravar o blefaroespasmo, tais como: claridade, estresse, fadiga, dirigir, ler e assistir televisão. Nas maiores séries, a maioria dos pacientes apresentam queixas de fotofobia ou irritação ocular que precede ou acompanha os espasmos palpebrais(1-2,10). Ao contrário, dormir, relaxar, falar, andar e o uso de colírios lubrificantes costumam melhorar os espasmos ${ }^{(1-3)}$. Alguns pacientes aprendem a evitar situações que agravam a condição, enquanto outros desenvolvem maneirismos que envolvem o uso de outros músculos inervados pelo nervo facial ou atos de concentração mental para diminuir a frequência e intensidade dos espasmos. Estes maneirismos incluem cantar, bocejar, tossir, abrir a boca, apertar o nariz, mascar chicletes, comer, falar continuamente, esfregar as pálpebras, cobrir um dos olhos, pressionar outras áreas da face, tocar piano ou resolver palavras-cruzadas ${ }^{(1,20)}$

Os espasmos palpebrais vão ficando cada vez mais frequentes e intensos e os pacientes podem se tornar funcionalmente cegos, socialmente reclusos e incapazes de trabalhar ou de cuidar de si próprios. Henderson ${ }^{(1)}$ classificou $12 \%$ dos pacientes de sua série como funcionalmente cegos. No estudo de Anderson et al. ${ }^{(2)}$, a progressão dos sintomas para um envolvimento debilitante ocorreu ao longo de semanas em 11\%, meses em 37\% e anos em 52\% dos pacientes.

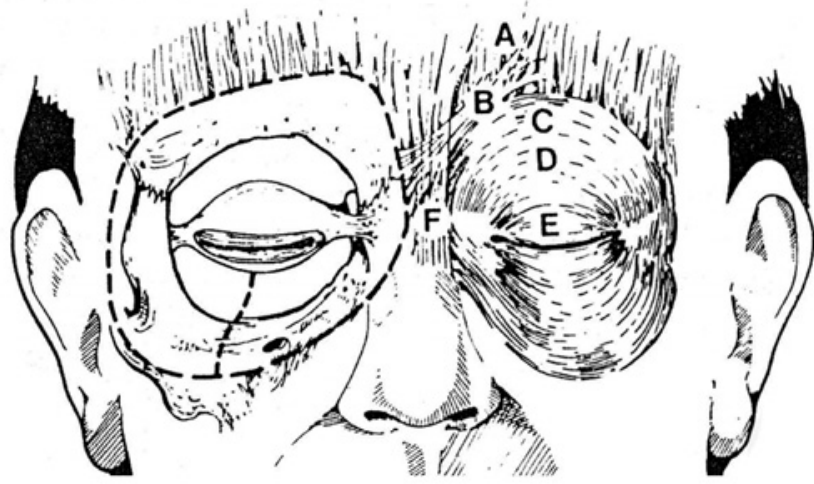

Figura 1. Músculos importantes para a função palpebral, intactos e identificados por letras. A) Frontal. B) Corrugador do supercílio. C) Orbicular, porção orbital.D) Orbicular, porção pré-septal.E) Orbicular, porção pré-tarsal. (F) Prócerus. Adaptado de Jordan et al. (3) $^{(3)}$
Um curso oscilante entre remissões e exacerbações é comum e durante a progressão pode haver o comprometimento dos músculos da região inferior da face ou de músculos cervicais(3).

Os músculos protratores (corrugador, prócerus e orbicular) são responsáveis pela oclusão palpebral; os músculos retratores (levantador da pálpebra superior, músculo de Müller e frontal) abrem as pálpebras superiores. A ação constante dessas forças oponentes, concomitante a tentativas manuais de abertura palpebral, causam diversas alterações palpebrais, tais como: dermatocálase, ptose palpebral ou dos supercílios, ectrópio ou entrópio das pálpebras inferiores. Essas deformidades palpebrais secundárias à doença podem ser funcionalmente tão debilitantes quanto os espasmos em $\mathrm{si}^{(3)}$

O comprometimento das atividades de vida diária dos pacientes pode ser importante. Cinquenta e oito por cento dos 1.653 pacientes que responderam ao questionário de Anderson et al.(2) declararam estar incapazes de dirigir veículos, $46 \%$ incapazes de ler, 41\% de assistir televisão, 29\% de trabalhar e $18 \%$ de sair de casa sozinhos. Apenas $14 \%$ dos pacientes com blefaroespasmo essencial afirmaram não apresentar comprometimento significante de suas atividades de vida diária.

\section{DIAGNÓSTICO DIFERENCIAL}

Avaliação oftalmológica completa e exame neurológico são importantes para se estabelecer o diagnóstico de blefaroespasmo essencial ${ }^{(3)}$. Espasmos palpebrais reflexos podem ser secundários a irritações corneanas presentes em pacientes com síndrome do olho seco, entrópio espástico, triquíase, blefarite, entre outras condições. Indivíduos com dor ocular ou fotofobia decorrente de quadros de uveíte anterior ou catarata subcapsular posterior também podem apresentar um blefaroespasmo reflexo.

Fasciculações do músculo orbicular, conhecidas como mioquimia, são geralmente unilaterais e temporárias, ocorrendo especialmente em períodos de estresse físico ou emocional, fadiga ou consumo excessivo de café(21).

No caso de fasciculações do músculo orbicular, que se iniciam na região periocular e gradualmente envolvem músculos inferiores da face, inervados pelo nervo facial, o diagnóstico é espasmo hemifacial. Esses espasmos são involuntários, agravados por estresse e podem persistir durante o sono ${ }^{(22)}$. Frequentemente há fraqueza dos músculos da face, achado não presente no blefaroespasmo, em que a força da musculatura facial é normal ou mesmo aumentada ${ }^{(3)}$. Assim como a neuralgia do trigêmio, o espasmo hemifacial é considerado uma síndrome de "loop" vascular, por ser resultante da compressão da emergência do nervo facial por um vaso sanguíneo com curso anormal(23). Embora muito menos comum, a compressão do nervo facial também pode ocorrer dentro do osso temporal, devido a presença de tumores ${ }^{(24)}$.

A apraxia de abertura palpebral é uma anormalidade motora caracterizada pela dificuldade em iniciar o movimento de elevação da pálpebra superior. Por ação do músculo frontal, ocorre elevação dos supercílios, sem que ocorra a abertura palpebral. Alguns autores acreditam que a apraxia seja o resultado de contrações persistentes, de baixa intensidade do músculo orbicular, que se opõe à ação do músculo levantador da pálpebra superior ${ }^{(25-26)}$.

Tique facial constitui diagnóstico de exclusão. Em contraste com o blefaroespasmo, está sob controle voluntário, não melhora com repouso e é geralmente observado em pacientes jovens ${ }^{(3)}$.

\section{TRATAMENTO}

\section{FARMACOTERAPIA}

Várias drogas têm sido testadas no manejo de pacientes com blefaroespasmo, com resultados limitados e temporários 
na maioria dos $\operatorname{casos}^{(27)}$. A lista inclui antipsicóticos, antidepressivos, ansiolíticos, estimulantes, sedativos, parassimpaticomiméticos, antimuscarínicos, inibidores da síntese de catecolaminas, anti-histamínicos, anticonvulsivantes, antiparkinsonianos, entre outras ${ }^{(3)}$.

No estudo de Anderson et al.(2), dos 1.653 pacientes com blefaroespasmo, 1.162 (70\%) tinham sido tratados com alguma medicação oral e apenas 43\% destes referiram ter experimentado alguma melhora. Entre estes, 52\% perceberam meIhora inferior a 50\%, 22\% notaram melhora entre 50\% e 75\%, $14 \%$ entre $75 \%$ e $90 \%$ e apenas $12 \%$ observaram melhora superior a $90 \%$.

\section{DenerVaÇão química do músculo orbicular}

Doxorrubicina é uma droga antimitótica e antimetabólica, com propriedades miotóxicas ${ }^{(28)}$. Wirtschafter e McLoon ${ }^{(29)}$ descreveram os resultados obtidos após aplicação de doxorrubicina no músculo orbicular de 18 pacientes com blefaroespasmo. Apenas 10 pacientes completaram o tratamento, sendo que 9 (90\%) desses foram considerados curados, permanecendo entre 1 a 6 anos (média=3 anos) sem a necessidade de tratamento adicional dos espasmos. As principais razões para a descontinuidade do tratamento, ocorrida em 8 (45\%) de 18 pacientes, foram uma esperança (injustificável, segundo os autores) de cura com apenas uma aplicação e pelas alterações cutâneas importantes em relação à redução dos espasmos após a primeira administração.

Trata-se de um método eficaz para o tratamento do blefaroespasmo, embora tenha o seu uso bastante limitado pelas reações cutâneas e seja empregado por poucos profissionais atualmente.

\section{TOXINA BOTULÍNICA}

O uso da toxina botulínica foi introduzido por $S \operatorname{cott}{ }^{(30)} \mathrm{em}$ 1980, como uma alternativa para o tratamento do estrabismo. Atualmente, a toxina é a terapia de escolha para pacientes com blefaroespasmo ${ }^{(31)}$.

Aplicações subcutâneas na pálpebra superior e inferior têm alto grau de aceitação por parte dos pacientes ${ }^{(3)}$. A maioria dos pacientes com blefaroespasmo essencial requer nova aplicação após 12 a 16 semanas ${ }^{(3,32-33)}$. Em nosso meio, Costa et al. ${ }^{(34)}$ avaliaram a eficácia desse tratamento por meio de questionários e 15 em 25 pacientes (60\%) com blefaroespasmo essencial ou síndrome de Meige referiram tempo de remissão entre 3 e 4 meses e 10 em 15 pacientes (40\%) disseram apresentar efeito por apenas 1 a 2 meses. Hsiung et al. ${ }^{(35)}$ estudando diversos tipos de distonias, observaram que os pacientes com blefaroespasmo são aqueles que mantêm as melhores respostas à toxina ao longo do tratamento, sendo que 33 em 36 (92\%) e 18 em 20 (90\%) pacientes apresentam boas respostas após 2 e 5 anos respectivamente.

Alguns autores referem que as primeiras aplicações trazem melhores resultados ${ }^{(3,32-33)}$. Após algumas aplicações, os pacientes requerem doses maiores, que têm menos efeito e duram menos tempo. Ainsworth e $\mathrm{Kraft}^{(33)}$ observaram que pacientes com blefaroespasmo necessitaram de um aumento de 50\% da dose nas primeiras 12 aplicações. Entretanto, o intervalo livre de sintomas não diminuiu significativamente ao longo do tratamento, embora tenha havido tendência a declínio discreto na duração do alívio. Essa alteração da dose pode ser necessária devido à formação de anticorpos circulantes neutralizadores da toxina botulínica, conforme postulado por diversos autores ${ }^{(36-38)}$.

A resistência à aplicação ao longo do tempo (resistência secundária) pode ser devida às suas propriedades imunológicas, que estimulam a produção de anticorpos, tornando os tratamentos adicionais ineficazes ${ }^{(36)}$. Essa imunogenicidade de- pende da dose por injeção, da aplicação de doses adicionais em curtos espaços de tempo, da dose acumulada e da frequência de injeções. Entretanto, a dose mínima exata e a frequência necessária para induzir a formação de anticorpos ainda é desconhecida ${ }^{(37)}$. Jankovic e Schwartz ${ }^{(38)}$ descreveram uma diferença significante na dose acumulada do tratamento, entre pacientes com anticorpos e sem anticorpos contra a toxina botulínica. De fato, Greene et al. ${ }^{(36)}$ observaram que pacientes resistentes à toxina receberam doses mais altas no início do tratamento ou aplicações adicionais em curto espaço de tempo, quando comparados com pacientes respondedores e os primeiros também tinham alta prevalência de anticorpos neutralizadores. A resposta ao tratamento foi perdida em 15,6 meses em média, mas a soropositividade foi documentada somente após cerca de 22,5 meses de tratamento. Aparentemente, injeções de pequenos volumes, com altas doses, que localizam a toxina são superiores aos tratamentos com grandes volumes e doses baixas, em que ocorre difusão da toxina para os músculos adjacentes. Esses achados foram confirmados por Jankovic et al. ${ }^{(38)}$, que demonstraram a associação entre altas doses e injeções frequentes com antigenicidade e aumento da produção de anticorpos neutralizadores da toxina.

A resistência primária à toxina botulínica (pacientes que não respondem ao tratamento, desde a primeira aplicação) é inferior a $10 \%$ dos $\operatorname{casos}^{(35)}$

Huang, Foster ${ }^{(39)}$ demonstraram que diversas medicações sistêmicas usadas cronicamente podem interferir no efeito terapêutico da toxina.

No estudo de Anderson et al. ${ }^{(2)}, 1.083$ pacientes (66\%) foram submetidos ao tratamento com toxina botulínica. Oitenta e seis por cento dos indivíduos referiram melhora dos espasmos. Entre esses, 24\% apresentaram melhora inferior a 50\%, 32\% entre 50 e $75 \%$ e $44 \%$ descreveram entre 75 e $90 \%$ de melhora dos sintomas. Curiosamente, nenhum paciente referiu melhora funcional superior a 90\% nesse estudo. Em relação à duração do efeito terapêutico, esse foi inferior a 2 semanas em $8 \%$ dos pacientes, entre 2 e 4 semanas em 5\%, 1 a 2 meses em 22\%, 3 a 4 meses em $44 \%$ e superior a 4 meses em $22 \%$ dos pacientes.

Os efeitos colaterais da toxina botulínica do tipo A são geralmente bem tolerados pela maioria dos pacientes ${ }^{(3,32)}$. Ptose transitória é a complicação mais frequente ${ }^{(31,35)}$.

Lagoftalmo e ceratite de exposição são comuns e geralmente duram até duas semanas. Lacrimejamento pode ser secundário a ceratite ou por falha da bomba lacrimal. Essas complicações podem ser tratadas com lubrificantes oculares ${ }^{(3)}$. Diplopia transitória, por envolvimento de músculos extraoculares, também pode ser observada ${ }^{(3,32)}$.

Ainda não se descreveu nenhum efeito sistêmico em função do uso da toxina botulínica, bem como não há relatos de anafilaxia ou óbito por uso dessa medicação.

\section{Tratamento CiRúrgico}

Pacientes com quadros debilitantes de blefaroespasmo e que não toleram ou não responderam bem ao tratamento com toxina botulínica são candidatos ao tratamento cirúrgico.

Os principais objetivos da cirurgia são: A) corrigir as alterações funcionais e eventualmente cosméticas que podem estar associadas (ptose de supercílios, ptose palpebral, dermatocalase, ectrópio, distopia cantal, etc.); B) reduzir a intensidade dos espasmos; C) diminuir a dosagem de toxina botulínica ou intervalo entre as aplicações; e, D) melhorar apraxia de abertura palpebral.

A miectomia originalmente descrita por Gillum e Anderson ${ }^{(40)}$ envolvia a completa remoção dos músculos protratores ao redor das pálpebras. Os músculos orbicular da pálpebra superior e inferior e sua extensão na região da rafe temporal, corrugador do supercílio e prócerus eram ressecados através de uma 
incisão sobre o supercílio. Entretanto, esse procedimento tinha alta morbidade, com linfedema e posições imprevisíveis das pálpebras no pós-operatório. Mais recentemente, preconiza-se apenas a realização da miectomia superior em um primeiro tempo, a fim de se prevenir o linfedema. A miectomia inferior tem sido reservada para os pacientes que persistem com espasmos importantes da pálpebra inferior e terço médio da face e deve ser realizada pelo menos seis meses após a primeira cirurgia(26).

No estudo de Anderson et al.(2), 330 pacientes (20\% da amostra) foram submetidos a miectomia superior. Metade desses pacientes foram também submetidos a miectomia inferior. Entre os pacientes operados, $88 \%$ referiram melhora. Vinte e sete por cento dos pacientes experimentaram menos de $50 \%$ de melhora, $21 \%$ referiram entre 50 e $75 \%$ de melhora, $29 \%$ entre 75 e $90 \%$ de melhora e $23 \%$ referiram melhora dos sintomas superior a 90\%. Dezoito por cento dos pacientes necessitaram de ressecções adicionais ou de uma revisão após a cirurgia. Trinta e oito por cento dos pacientes precisaram de aplicações de toxina botulínica após a cirurgia (segundo o autor, provavelmente a maioria destes pacientes eram provenientes do grupo não submetido a miectomia inferior).

Anderson et al. ${ }^{(2)}$ enumera as complicações ocorridas em mais de 500 miectomias realizadas: infecção, hematoma ou hemorragia, queda dos pelos da sobrancelha, perda de pele (necessitando de enxerto), ptose, retração de pálpebra superior e inferior, triquíase e deformidades de canto. O autor ainda pondera que a maioria das complicações foram pequenas e passíveis de correção, excetuando-se alguns casos de deformidade permanente. Além disso, apesar do porte da cirurgia e da idade avançada de muitos pacientes, ele não refere nenhum caso de óbito ou de deficiência permanente após o procedimento.

Linfedema crônico é a complicação mais difícil de lidar, podendo durar mais de um ano e é muito mais intenso quando se realiza a miectomia superior e inferior no mesmo tempo cirúrgico $(2-3,27)$

Patel ${ }^{27)}$ descreveu uma técnica de miectomia que associa endoscopia à técnica aberta convencional, evitando-se a incisão sobre o supercílio, na tentativa de se minimizar os efeitos colaterais, como a anestesia regional por dano do nervo supraorbital.

\section{REFERÊNCIAS}

1. Henderson JW. Essential blepharospasm. Trans Am Ophthalmol Soc. 1956;54:453-520.

2. Anderson RL, Patel BC, Mantém JB, Jordan DR. Blepharospasm: past, present, and future. Ophthal Plast Reconstr Surg. 1998;14(5):305-17.

3. Jordan DR, Patrinely JR, Anderson RL, Thiese SM. Essential blepharospasm and related dystonias. Surv Ophthalmol. 1989;34(2):123-32. Review.

4. Defazio G, Livrea P. Epidemiology of primary blepharospasm. Mov Disord. 2002; 17(1):7-12.

5. Cossu G, Mereu A, Deriu M, Melisa M, Molari A, Melis G, et al. Prevalence of primary blepharospasm in Sardinia, Italy: a service-based survey. Mov Disord. 2006;21(11): 2005-8.

6. Nutt JG, Muenter MD, Aronson A, Kurland LT, Melton $L 3^{\text {rd }}$. Epidemiology of focal and generalized dystonia in Rochester, Minnesota. Mov Disord. 1988;3(3):188-94.

7. Bradley EA, Hodge DO, Bartley GB. Benign essential blepharospasm among residents of Olmsted County, Minnesota, 1976 to 1995: an epidemiologic study. Ophthal Plast Reconstr Surg. 2003;19(3):177-81.

8. Defazio G, Martino D, Abbruzzese G, Girlanda P, Tinazzi M, Fabbrini G, et al. Influence of coffee drinking and cigarette smoking on the risk of primary late onset blepharospasm: evidence from a multicentre case control study. J Neurol Neurosurg Psychiatry. 2007:78(8): 877-9.

9. McCann JD, Gauther M, Morschbacher R, Goldberg RA, Anderson RL, Fine PG, et al. A novel mechanism for benign essential blepharospasm. Ophthal Plast Reconstr Surg. 1999;15(6):384-9.

10. Elston JS, Marsden CD, Grandas F, Quinn NP. The significance of ophthalmological symptoms in idiopathic blepharospasm. Eye (Lond). 1988;2 (Pt 4):435-9.
11. Esmaeli-Gutstein B, Nahmias C, Thompson M, Kazdan M, Harvey J. Positron emission tomography in patients with benign essential blepharospasm. Ophthal Plast Reconstr Surg. 1999;15(1):23-7.

12. Schmidt $K E$, Linden $D E$, Goebel $R$, Zanella FE, Lanfermann $H$, Zubcov AA. Striatal activation during blepharospasm revealed by fMRI. Neurology. 2003;60(11):1738-43. Comment in: Neurology. 2003;60(11):1732-3.

13. Baker RS, Andersen AH, Morecraft RJ, Smith CD. A functional magnetic resonance imaging study in patients with benign essential blepharospasm. J Neuroophthalmol. 2003;23(1):11-5

14. Grandas F, Lopez-Manzanares L, Traba A. Transient blepharospasm secondary to unilateral striatal infarction. Mov Disord. 2004;19(9):1100-2.

15. Defazio G, Livrea P, Guanti G, Lepore V, Ferrari E. Genetic contribution to idiopathic adult-onset blepharospasm and cranial-cervical dystonia. Eur Neurol. 1993; 33(5):345-50.

16. Defazio G, Martino D, Aniello MS, Masi G, Abbruzzese G, Lamberti S, et al. A family study on primary blepharospasm. J Neurol Neurosurg Psychiatry. 2006;77(2):252-4.

17. Defazio G, Brancatti F, Valente EM, Caputo V, Pizzuti A, Martino D, et al. Familial blepharospasm is inherited as an autosomal dominant trait and relates to a novel unassigned gene. Mov Disord. 2003:18(2):207-12.

18. Johnson LN, Lapour RW, Johnson GM, Johnson PJ, Madsen RW, Hackley SA. Closely spaced stressful life events precede the onset of benign essential blepharospasm and hemifacial spasm. J Neuroophthalmol. 2007;27(4):275-80.

19. Costa PG, Cardoso IP, Saraiva FP, Raiza AC, Tanaka LK, Matayoshi S. [Lacrimal film evaluation of patients with facial dystonia during botulinum toxin type A treatment]. Arq Bras Oftalmol. 2006;69(3):319-22. Portuguese.

20. Jankovic J, Ford J. Blepharospasm and orofacial-cervical dystonia: clinical and pharmacological findings in 100 patients. Ann Neurol. 1983;13(4):402-11.

21. Boghen DR. Disorders of facial motor function. Curr Opin Ophthalmol. 1996;7(6): 48-52.

22. Wang A, Jankovic J. Hemifacial spasm: clinical findings and treatment. Muscle Nerve. 1998:21(12):1740-7.

23. Port JD. Advanced magnetic resonance imaging techniques for patients with hemifacial spasm. Ophthal Plast Reconstr Surg. 2002;18(1):72-4.

24. Sprik C, Wirtschafter JD. Hemifacial spasm due to intracranial tumor. An international survey of botulinum toxin investigators. Ophthalmology. 1988;95(8):1042-5.

25. Tozlovanu V, Forget R, lancu A, Boghen D. Prolonged orbicularis oculi activity: a major factor in apraxia of lid opening. Neurology. 2001;57(6):1013-8.

26. Georgescu D, Vagefi MR, McMullan TF, McCVann JD, Anderson RL. Upper eyelid myectomy in blepharospasm with associated apraxia of lid opening. Am J Ophthalmol. 2008;145(3):541-7.

27. Patel BC. Surgical management of essential blepharospasm. Otolaryngol Clin North Am. 2005;38(5):1075-98

28. Baker L, Wirtschafter JD. Experimental doxorubicin myopathy. A permanent treatment for eyelid spasms? Arch Ophthalmol. 1987;105(9):1265-8.

29. Wirtschafter JD, McLoon LK. Long-term efficacy of local doxorubicin chemomyectomy in patients with blepharospasm and hemifacial spasm. Ophthalmology. 1998; 105(2):342-6.

30. Scott AB. Botulinum toxin injection into extraocular muscles as an alternative to strabismus surgery. Ophthalmology. 1980;87(10):1044-9.

31. Scott AB, Kennedy RA, Stubbs HA. Botulinum A toxin injection as a treatment for blepharospasm. Arch Ophthalmol. 1985;103(3):347-50

32. Snir M, Weinberger D, Bourla D, Kristal-Shalit O, Dotan G, Axer-Siegel R. Quantitative changes in botulinum toxin a treatment over time in patients with essential blepharospasm and idiopathic hemifacial spasm. Am J Ophthalmol. 2003:136(1):99-105.

33. Ainsworth JR, Kraft SP. Long-term changes in duration of relief with botulinum toxin treatment of essential blepharospasm and hemifacial spasm. Ophthalmology. 1995:102(12):2036-40.

34. Costa PG, Aoki L, Saraiva FP, Matayoshi S. [Botulinum toxin in the treatment of facial dystonia: evaluation of its efficacy and patients' satisfaction along the treatment]. Arq Bras Oftalmol. 2005;68(4):471-4. Portuguese

35. Hsiung GY, Das SK, Ranawaya R, Lafontaine AL, Scuchowersky O. Long-term efficacy of botulinum toxin $A$ in treatment of various movement disorders over a 10-year period. Mov Disord. 2002;17(6):1288-93. Comment in: Mov Disord. 2003;18(9):1080-1.

36. Greene P, Fahn S, Diamond B. Development of resistance to botulinum toxin type A in patients with torticollis. Mov Disord. 1994;9(2):213-7.

37. Eleopra R, Tugnoli V, de Grandis D. The variability in the clinical effect induced by botulinum toxin type A: the role of muscle activity in humans. Mov Disord. 1997; 12(1):89-94.

38. Jankovic J, Schwartz K. Response and immunoresistance to botulinum toxin injections. Neurology. 1995;45(9):1743-6.

39. Huang W, Foster JA, Rogachefsky AS. Pharmacology of botulinum toxin. J Am Acad Dermatol. 2000;43(2 Pt 1):249-59.

40. Gillum WN, Anderson RL. Blepharospasm surgery. An anatomical approach. Arch Ophthalmol. 1981;99(6):1056-62. 